\title{
The Effect Analysis of Geometry and Load Parameters on Contact Stiffness of Sprag One-way Clutch
}

\author{
Zhi-Hui LIU ${ }^{1,2,3}$, Hong-Zhi YAN ${ }^{1,3, a}$, Yu-Ming CAO ${ }^{1,3}$, Yu-Qing LAI ${ }^{2}$ \\ ${ }^{1}$ The State Key Laboratory of High-performance and Complex Manufacturing, Central South \\ University, Changsha 410083, China \\ ${ }^{2}$ Department of Mechanical and Energy Engineering, Shaoyang University, Shaoyang 422000, \\ China \\ ${ }^{3}$ College of Mechanical and Electrical Engineering, Central South University, Changsha 410083, \\ China
}

Keywords: One-way clutch, Hertz contact theory, Normal contact stiffness, Sprag, Newton iterative method.

\begin{abstract}
The wedge-raceway contact mechanical model, considering centrifugal force of wedge and the eccentricity of the inner ring and the outer ring of the one-way clutch, is established based on Hertz contact theory. The normal contact stiffness of the sprag one-way clutch is calculated by Newton iterative method.The influence of the structure parameters including the eccentricity of the inner ring and the outer ring, labeled as e, the number of the sprags, labeled as $\mathrm{Z}$, the difference of the radius between the inner race of the outer ring and the outer race of the inner ring, labeled as $J$, and the working condition of the clutch including the working torque labeled as $\mathrm{T}$ and the working speed labeled as $n$, to the contact stiffness of the clutch has been analyzed. The results show that the smaller e, the smaller $\mathrm{J}$, the much more number of $\mathrm{Z}$ and the higher torque $\mathrm{T}$ will result the bigger stiffness, and the influence of the working speed labeled as $n$ can be negligible.
\end{abstract}

\section{Introduction}

The gear-sprag clutch system is one of the most important systems, and the sprag clutch's proper performance got a direct effect on the main and accessory drive chain of helicopter, the transmission between starter and motor of fixed-wing aircraft. The vertical, transverse and torsional vibration of gear-sprag clutch system is strongly related to its natural frequency, including stiffness, damping, and components' inertial masses 1-2]. The analytical calculation of clutch's stiffness is in order to determine the root cause of increased stiffness, finally decreasing the vibration of the gear-sprag clutch system.

In the present paper, there is a special attention to the effect of clutch's stiffness on the vibration of mechanic systems, such as belt-pulley system, gear-clutch system and so on. Zhu et al. [3] used a two-degree of freedom model to examine the effect of the clutch stiffness on the non-linear dynamics of a two-pulley belt system with a one-way clutch. Hu et al. [4] studied the effects of the clutch's stiffness on the nonlinear steady-state response of a belt-drive system with a one-way clutch, and had a conclusion that one-way clutch not only decreases the resonance amplitude of the driven pulley and shaft's rotational vibration, but also reduces the resonance region of the belt's transverse vibration. Hu et al. [5] also presented the stable steady-state periodic response of a twopulley belt drive system coupled with one-way clutch under double excitation for the first time. Cheon [6] verified the effect of the clutch's stiffness on the nonlinear behavior of a paired spur gear system with a one-way clutch for the first time.

As far as I know there is only little literature available on the theoretical calculation and experimental test of clutch's stiffness. The overrunning clutch stiffness analysis is essentially based on the static analysis of Hertz contact deformation theory [7-10], irrespective of the influence of centrifugal force of cylindrical rollers or wedges, manufacturing error, and only torsional stiffness is analyzed. Sprag clutch generally works under the condition of high speed and heavy load, and there exists installation error and other inevitable manufacturing errors. Therefore, it is necessary to make 
an in-depth analysis of the influence on the stiffness of clutch from structural parameters, working conditions and manufacturing errors.

The wedge-raceway contact mechanical model, considering the eccentricity of the inner ring and the outer ring of the one-way clutch, is established based on Hertz contact theory. Using Newton iterative method the contact stiffness of the sprag one-way clutch is calculated. He normal contact stiffness is calculated by Newton iterative method. The influence of the structure parameters including the eccentricity of the inner ring and the outer ring, labeled as e, the number of the sprags, labeled as $\mathrm{Z}$, the difference of the radius between the inner race of the outer ring and the outer race of the inner ring, labeled as $\mathrm{J}$, and the working condition of the clutch including the working torque labeled as $\mathrm{T}$ and the working speed labeled as $\mathrm{n}$, on the contact stiffness of the clutch has been analyzed.

\section{Basic Assuptions}

When calculating the stiffness of the clutch, the following assumptions are made:

1) The incline of the inner ring, wedge, and outer ring is ignored. The inner ring, wedge, and the outer ring move within mutually parallel planes;

2) Only considering the deformation of the inner ring and outer ring contact surface, the wedge is considered as a rigid body;

3) The relationship between the elastic contact force and contact deformation of the wedge block, the inner ring and the outer ring contact surface meets the Hertz contact theory;

4) Compared to the Hertz contact force, the force between the wedge and the spring, the wedge block and the retainer can be negligible;

5) The wedge is uniformly distributed along the circumferential direction of the outer ring.

\section{Stiffness Calculation Model}

\section{The Geometric Model of Sprag One-Way Considering the Eccentricity of the Inner Ring and the Outer Ring.}

There inevitably exist eccentricity between inner ring and outer ring, as shown in Fig. 1, when sprag one-way clutch is assembled. The inner strut angle $\alpha(\mathrm{ij})$, which is the angle between the normal through the contact point on outer race of inner ring and the line, connecting the two contact points between the sprag and the outer ring and the inner ring, the outer strut angle $\alpha(0 j)$, which is the angle between the normal through the contact point on inner race of the outer ring and the line, connecting the two contact points between the sprag and the outer ring and the inner ring, and the loads which are applied on each sprags are different. The solid line in Fig.1 stands for the connection diagram between the sprags and the inner ring and the undeformed outer ring, and the dashed line stands for that between the sprags and the inner ring and the deformed outer ring. The point $\mathrm{P}_{\mathrm{j}}$ is the jth contact point between the outer cam of sprag and the deformed inner race of outer ring, and the point $\mathrm{Q}_{\mathrm{j}}$ is the $\mathrm{jth}$ contact point between the inner cam and the deformed outer race of the inner ring. It is assumed that the sprag located in vertical axis are defined the first one $(j=1)$. The outer cam of logarithmic type sprag is circular arc surface and the inner cam profile is logarithmic curve. The center of the circular arc and the pole of the logarithmic curve are designed as the same point. When sprags are being wedged between the inner ring and the outer ring, the inner race of the outer ring and the outer race of the inner ring will be deformed, and the sprags will do relatively planar motion relative to the initial position, rotating around its center of gravity. The normal through the point $\mathrm{P}_{\mathrm{j}}$, must be through the pole of the logarithmic curve, the initial contact point and the circle of the outer ring $\mathrm{O}$, and the normal through the point $\mathrm{Q}_{\mathrm{j}}$ must be through the circle of the inner ring $\mathrm{O}^{\prime \prime}$. When the sprags are rotating, leading to the deformation of the outer ring and the inner ring, the pole will be offset upward the deformation of outer ring $\delta_{\mathrm{oj}}$ along the direction of the normal through the point $\mathrm{P}_{\mathrm{j}} . \delta_{\mathrm{oj}}, \delta_{\mathrm{ij}}$ stand for the deformation of outer ring and inner ring respectively, which the $\mathrm{jth}$ 
sprag contact with. The subscript $\mathrm{i}, \mathrm{o}, \mathrm{j}$ represent the inner ring, the outer ring and the $\mathrm{jth}$ sprag separately.

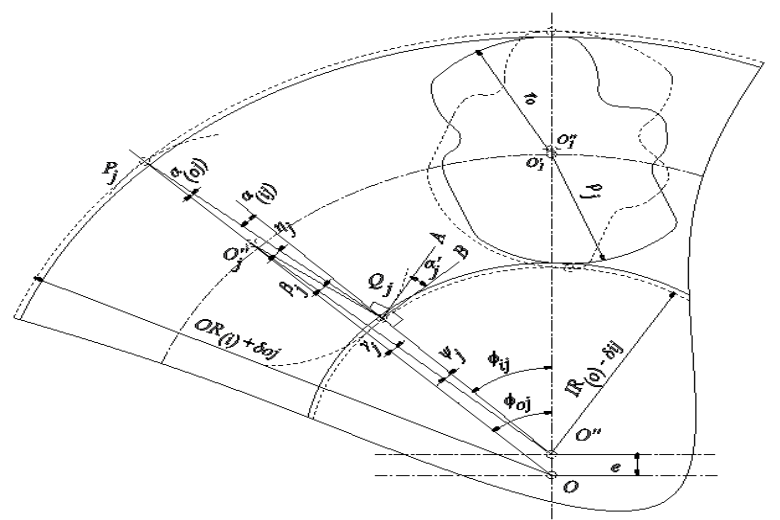

1. Solid line stands for overrunning state. 2. Dash line stands for engaged state.

Figure 1. The geometric model and load deformation diagram of the sprag one-way clutch.

\section{The Calculation of Normal Angle of Sprag One-Way Clutch}

Referring to Fig. 1, it is assumed that the sprags are evenly spaced apart along the inner ring circumferential direction when the sprag one-way clutch is assembled.

$$
\phi_{o j}=2 \pi(j-1) / Z
$$

Фoj iscalledouter azimuth angle, which is between the line through outer ring central point $\mathrm{O}$ and the Jth sprag contact point $\mathrm{Pj}$ with deformed outer ring and the normal direction of $\mathrm{Y}$ axis. It can be obtained by the method of sine theorem in triangle $\mathrm{OO}^{\prime \prime} \mathrm{O}_{j}$ ".

$$
\gamma_{j}=\arcsin \left(\frac{e \sin \phi_{o j}}{\sqrt{\left(O R_{(i)}+\delta_{o j}-r_{o}\right)^{2}+e^{2}-2\left(O R_{(i)}+\delta_{o j}-r_{o}\right) e \cos \phi_{o j}}}\right)
$$

Where $e$ is the eccentricity of the inner ring and outer ring. The deformation $\delta_{\mathrm{oj}}$ is the outer ring deformation, which is contacted with the jth sprag. In the equation, OR(i) is the radius of inner race of outer ring, and $r_{o}$ is the radius of outer cam of sprag. Eq. 3 can be obtained by reference 2 .

$$
\delta_{o j}=0.614\left(\frac{2 O R_{(i)}-r_{o}}{O R_{(i)} r_{o}}\right)^{\frac{1}{3}}\left(\frac{F_{n j(o)}}{E}\right)^{\frac{2}{3}}
$$

Where $F_{n j}(o)$ is the is the normal contact force between the jth sprag and the inner race of outer ring.

As the line $\mathrm{Q}_{\mathrm{j}} \mathrm{A}$ is perpendicular to the pole $\mathrm{O}_{\mathrm{j}}$ ", and the line $\mathrm{Q}_{\mathrm{j}} \mathrm{B}$ is perpendicular to the line $\mathrm{O}^{\prime \prime} \mathrm{Q}_{\mathrm{j}}$. The curve of the sprag inner cam is Logarithmic curve.It can be obtained by the sine theorem in triangle $\Delta \mathrm{O}^{\prime \prime} \mathrm{O}_{\mathrm{j}} " \mathrm{Q}_{\mathrm{j}}$, according to characteristics of the logarithmic curve[11].

$$
\begin{gathered}
\beta_{j}=\arcsin \frac{\left(I R_{(o)}-\delta_{i j}\right) \sin \alpha_{j}^{\prime}}{\sqrt{\left(O R_{(i)}+\delta_{o j}-r_{o}\right)^{2}+e^{2}-2\left(O R_{(i)}+\delta_{o j}-r_{o}\right) e \cos \phi_{o j}}} \\
\psi_{j}=\alpha^{\prime}{ }_{j}-\beta_{j}=\arctan m-\beta_{j}
\end{gathered}
$$

Where $\operatorname{IR}(0)$ is the radius of outer race of inner ring, and $F_{n j}(i)$ is the normal contact force between the jth sprag and the outer race of the inner ring. It is similarly that the deformation $\delta_{\mathrm{ij}}$ is the inner ring deformation, which is contacted with the jth sprag. Eq. 6 can be similarly obtained by reference 3 . 


$$
\delta_{i j}=0.614\left(\frac{2 I R_{(o)}+\rho_{j} \sqrt{1+m^{2}}}{I R_{(o)} \rho_{j} \sqrt{1+m^{2}}}\right)^{\frac{1}{3}}\left(\frac{F_{n j(i)}}{E}\right)^{\frac{2}{3}}
$$

Where $\mathrm{m}$ is parameter of logarithmic curve and $\mathrm{E}$ is the modulus of elasticity. It can be obtained by the property of supplementary angle in triangle $\mathrm{OO}^{\prime \prime} \mathrm{O}_{\mathrm{j}}$ ".

$$
\phi_{i j}=\phi_{o j}+\gamma_{j}-\psi_{j}
$$

Similarly, $\Phi_{\mathrm{ij}}$ iscalledinner azimuth angle, which is between the line through inner ring central point $\mathrm{O}^{\prime}$ and the Jth sprag contact point $\mathrm{Q}_{\mathrm{j}}$ with deformed inner ring and the normal direction of $\mathrm{Y}$ axis.

\section{The Calculation of Normal Contact Force of Sprag One-Way Clutch}

Fig. 2 shows the force analysis of sprag. The outer strut angle $\alpha$ (oj)is the angle between the normal through the contact point on inner race of the outer ring and the line, connecting the two contact points between the jth sprag and the outer ring and the inner ring, and the inner strut angle $\alpha(\mathrm{ij})$ is the angle between the normal through the contact point on outer race of the inner ring and the line, connecting the two contact points between the jth sprag and the outer ring and the inner ring. $F_{w j}$ is the centrifugal force of sprag in high-speed movement. $\mathrm{T}$ is the external torque applied on the inner ring.

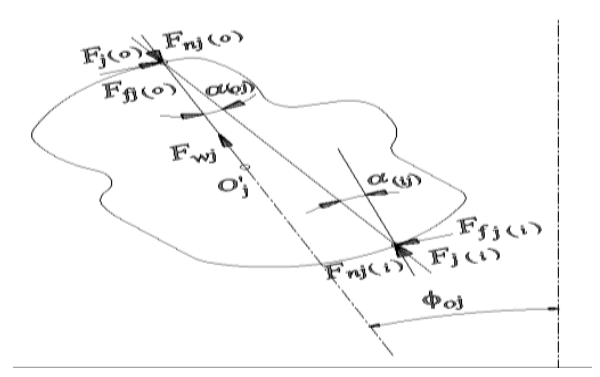

Figure 2. The force analysis of sprag.

It can be obtained by the sine theorem in triangle $\mathrm{P}_{\mathrm{j}} \mathrm{O}_{\mathrm{j}}{ }^{\prime \prime} \mathrm{Q}_{\mathrm{j}}$, referring to Fig. 1 .

$$
\begin{gathered}
\rho_{j}=\frac{\left(I R_{(o)}-\delta_{i j}\right) \sin \psi_{j}}{\sin \beta_{j}} \\
\eta_{j}=\arcsin \left(\frac{r_{o} \sin \left(\beta_{j}+\gamma_{j}\right)}{\sqrt{r_{o}^{2}+\rho_{j}{ }^{2}+2 r_{o} \rho_{j} \cos \left(\beta_{j}+\gamma_{j}\right)}}\right)
\end{gathered}
$$

On referring to Fig. 1, the straight line $\mathrm{O}^{\prime \prime} \mathrm{Q}_{j}$ is perpendicular to the straight line $\mathrm{Q}_{j} \mathrm{~B}$, and the straight line $\mathrm{Q}_{\mathrm{j}} \mathrm{O}_{\mathrm{j}}$ " is perpendicular to the straight line $\mathrm{Q}_{\mathrm{j}} \mathrm{A}$. The curve of the sprag inner cam is Logarithmic curve. Eq. 1 can be obtained by referring to literature [11].

$$
\alpha_{(i j)}=\alpha_{j}{ }^{\prime}-\eta_{j}=\arctan m-\eta_{j}
$$

The outer strut angle of the jth sprag can be calculated by the angle relationship of triangle.

$$
\alpha_{(o j)}=\beta_{j}+\gamma_{j}-\eta_{j}
$$

As shown in Fig. 2, the following formula can be obtained according to the force balance.

$$
F_{w j}=m_{s} R_{w j}\left(\frac{2 \pi n_{w}}{60}\right)^{2}
$$




$$
\begin{aligned}
& F_{n j(o)}=F_{w j}+F_{n j(i)} \cos \alpha_{(o j)} / \cos \alpha_{(i j)} \\
& \sum_{j=1}^{Z} F_{n j(o)} \tan \alpha_{(o j)}\left(O R_{(i)}+\delta_{o j}\right)=T
\end{aligned}
$$

Where $m_{s}$ is the mass of the sprag, $R_{w j}$ is the distance between the sprag center and the outer ring center, and $\mathrm{n}_{\mathrm{w}}$ is the rotation speed of sprag one-way clutch.

\section{The Calculation Model of Normal Contact Stiffness with Eccentricity $\mathbf{E}$ between the Inner Ring and Outer Ring}

Fig. 3 shows that the radial component in the $\mathrm{Y}$ axis of the contact stiffness $\mathrm{k}_{\mathrm{oj}}$ between the jth sprag and the inner race of outer ring and the contact stiffness $k_{\mathrm{ij}}$ between the jth sprag and the outer race of inner ring is respectively.

Eq. 15 and Eq. 16 can be obtained by using operations of partial derivatives on the normal contact deformation $\delta_{\mathrm{oj}}$ and $\delta_{\mathrm{ij}}$ in Eq. 3 and Eq. 6 respectively [12-14].

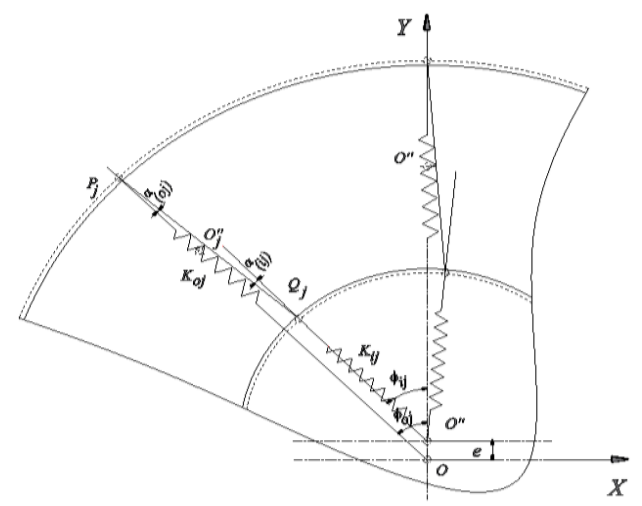

1. Solid line stands for overrunning state. 2. Dash line stands for engaged state.

Figure 3 . The contact stiffness between the wedge and the inner ring and outer ring.

$$
\begin{aligned}
& K_{o j}=\frac{d F_{n j(o)}}{d \delta_{o j}}=2.44\left(\frac{2 O R_{(i)}-r_{o}}{E^{2} O R_{(i)} r_{o}}\right)^{-\frac{1}{3}}\left(F_{n j(o)}\right)^{\frac{1}{3}} \\
& K_{i j}=\frac{d F_{n j(i)}}{d \delta_{i j}}=2.44\left(\frac{2 I R_{(o)}+\rho_{j} \sqrt{1+m^{2}}}{E^{2} I R_{(o)} \rho_{j} \sqrt{1+m^{2}}}\right)^{-\frac{1}{3}}\left(F_{n j(i)}\right)^{\frac{1}{3}}
\end{aligned}
$$

Fig. 3 shows that the radial component in the $\mathrm{Y}$ axis of the contact stiffness $\mathrm{k}_{\mathrm{oj}}$ between the jth sprag and the inner race of outer ring and the contact stiffness $\mathrm{k}_{\mathrm{ij}}$ between the $\mathrm{jth}$ sprag and the outer race of inner ring is respectively.

$$
\begin{aligned}
& K_{y o j}=K_{o j}\left|\cos \phi_{o j}\right| \\
& K_{y i j}=K_{i j}\left|\cos \phi_{i j}\right|
\end{aligned}
$$

As mentioned above, $\Phi_{\mathrm{ij}}$ is the angle between normal through the contact point $\mathrm{Q}_{\mathrm{j}}$, which is between the jth sprag and the inner ring, and the $\mathrm{Y}$ axis. $\Phi_{\mathrm{oj}}$ is the angle between the normal through the contact point $\mathrm{P}_{\mathrm{j}}$, which is between the jth sprag and the outer ring, and the $\mathrm{Y}$ axis. Both of those two angles are called azimuth Angle. The total radial component in the $\mathrm{Y}$ axis direction of 
the contact stiffness $\mathrm{k}_{\mathrm{ij}}$ and the contact stiffness $\mathrm{k}_{\mathrm{oj}}$ can be obtained with the principle of the spring in series.

$$
K_{y j}=\frac{K_{y i j} K_{y o j}}{K_{y i j}+K_{y o j}}
$$

The total radial component in the $\mathrm{Y}$ axis direction of all sprags contact stiffness can be obtained by the principle of the spring in parallel.

$$
K_{y}=\sum_{j=1}^{Z} K_{y j}
$$

The total radial component in the $\mathrm{X}$ axis direction of all sprags contact stiffness can be obtained in the same method.

$$
K_{x}=\sum_{j=1}^{Z} K_{x j}=\sum_{j=1}^{Z} \frac{K_{x i j} K_{x o j}}{K_{x i j}+K_{x o j}}=\sum_{j=1}^{Z} \frac{K_{\mathrm{ij}}\left|\sin \phi_{i j}\right| K_{o j}\left|\sin \phi_{o j}\right|}{K_{i j}\left|\sin \phi_{i j}\right|+K_{o j}\left|\sin \phi_{o j}\right|}
$$

It is obviously that the calculation of the total radial stiffness is statically indeterminate problem. Without considering the bending deformation of the inner ring and outer ring, the following formula can be increased with the condition of deformation coordination at $\Phi_{\mathrm{oj}}$ and $\Phi_{\mathrm{ij}}$.

$$
\begin{gathered}
\delta_{(o j)}=\delta_{o 1}\left|\cos \phi_{o j}\right| \\
\delta_{(i j)}=\delta_{i 1}\left|\cos \phi_{i j}\right| /\left|\cos \phi_{i 1}\right|
\end{gathered}
$$

As shown in Fig. $1, \delta_{\mathrm{o} 1}$ is the normal deformation between the sprag in $\mathrm{Y}$ axis and the inner race of the outer ring, $\delta_{\mathrm{i} 1}$ is the normal deformation between the sprag in $\mathrm{Y}$ axis and the outer race of inner ring, and $\Phi_{i 1}$ is the angle between the normal through the contact point on the outer race of inner ring and $\mathrm{Y}$ axis.

\section{Calculation and Result Analysis}

The main parameters of sprag clutch are shown in Table 1. The calculation results are shown in Fig.4 to Fig.9, using the Newton iteration method for solving the above equations.

Table 1. The key parameters of sprag one-way clutch for $\mathrm{J}=9.5 \mathrm{~mm}$.

\begin{tabular}{|c|c|}
\hline Parameter & Data \\
\hline Inner race of outer ring, $O R_{(\mathrm{i})} / \mathrm{mm}$ & 45.5995 \\
\hline Outer race of inner ring, $I R_{(\mathrm{o})} / \mathrm{mm}$ & 36.106 \\
\hline Outer cam radius of sprag, $r_{\mathrm{o}} / \mathrm{mm}$ & 4.9233 \\
\hline Mass of sprag, $m_{\mathrm{s}} / \mathrm{g}$ & 9.427 \\
\hline Eccentricity of inner and outer ring centers, $e / \mathrm{mm}$ & 0.01 \\
\hline Transfer torque, $T / \mathrm{N} . \mathrm{m}$ & 1326.25 \\
\hline Elastic modulus, $E / \mathrm{Gpa}$ & 206 \\
\hline Number of sprags, $Z$ & 33 \\
\hline Logarithmic curve parameter, $m$ & 0.1036 \\
\hline Revolution, $r / \mathrm{min}$ & 7200 \\
\hline
\end{tabular}

Figure 4 shows the influence of eccentricity between inner ring and outer ring of sprag one-way clutch on its normal contact stiffness. It is shown that the normal contact stiffness in $\mathrm{Y}$ axis 
direction is greater than that in $\mathrm{X}$ axis direction. With the increase of eccentricity, the normal contact stiffness in two axis decreases monotonously. When the eccentricity is increased from $0.01 \mathrm{~mm}$ to $0.1 \mathrm{~mm}$, the normal contact stiffness in $\mathrm{X}$ axis direction will be decreased by $0.45 \%$, and the normal contact stiffness in $\mathrm{Y}$ axis direction will be decreased by $0.71 \%$.

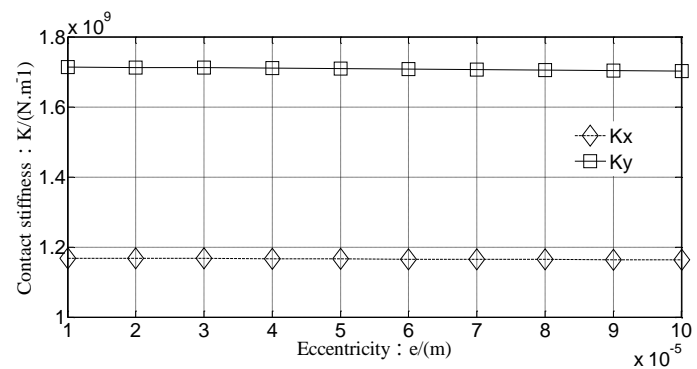

Figure 4. The change rule of radial contact stiffness under different eccentricity e.

Figure 5 shows the influence of the number of the sprags on the normal contact stiffness of sprag one-way clutch. With the number of sprags, the normal contact stiffness in $\mathrm{X}$ axis and $\mathrm{Y}$ axis direction increase monotonously. When the number of sprags is increased from 31 to 35, the normal contact stiffness in $\mathrm{X}$ axis direction is increased by $2.31 \%$, and the normal contact stiffness in $\mathrm{Y}$ axis direction is increased by $3.2 \%$.

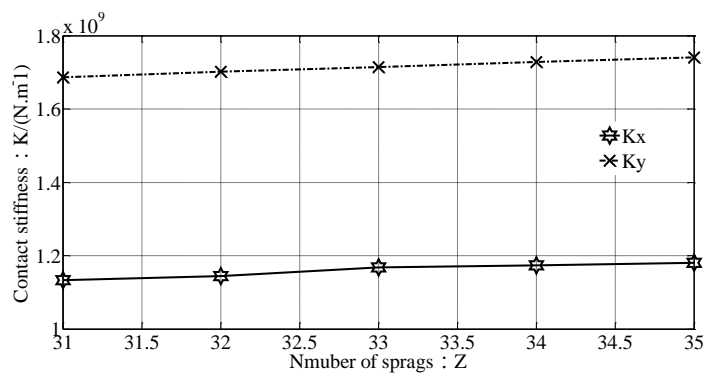

Figure 5. The change rule of radial contact stiffness under different number of sprags $\mathrm{Z}$.

For sprag clutch, there are four kinds of specification $\mathrm{J}$, which is defined as the radius difference between the inner race of the outer ring and the outer race of the inner ring, as shown in Figure 6 . With the increase of specification $\mathrm{J}$, the normal contact stiffness in $\mathrm{X}$ and $\mathrm{Y}$ axis direction decrease monotonously. The difference of the normal contact stiffness in $\mathrm{X}$ axis direction between the maximum value and the minimum value is $2.76 \%$, and the difference in $\mathrm{Y}$ axis direction between the maximum value and the minimum value is $6.21 \%$.

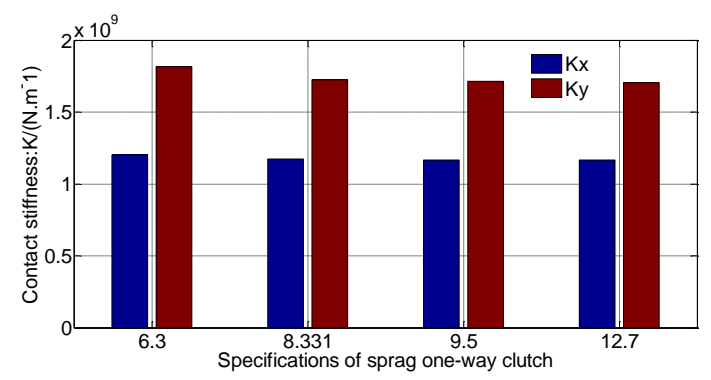

Figure 6. The change rule of radial contact stiffness under different specification of sprag one-way clutch J.

The normal loads applied on each sprag between the inner race of outer ring and the outer race of inner ring will no longer be equal each other, when the center lines of inner ring and outer ring are misalignment. It is shown in Figure 7 that the distribution of the normal load applied on each sprag is changed with the azimuth Angle. It can be concluded that every sprag are applied on normal 
contact load, where the load applied on the sprag locating in the top and bottom of $\mathrm{Y}$ axis direction is the maximum value and the load applied on the sprag locating in $\mathrm{X}$ axis direction is the minimum value.

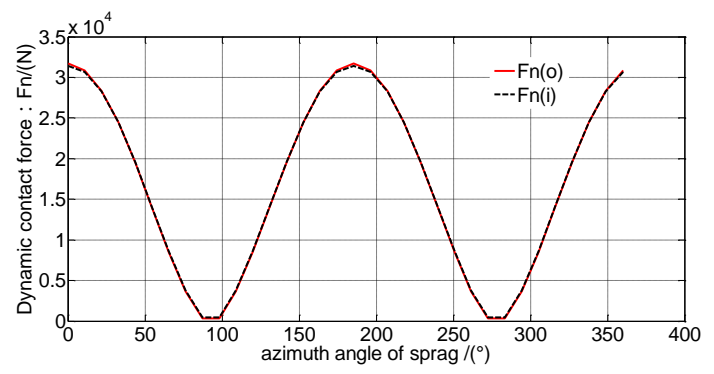

Figure 7. The change rule of dynamic contact force under different azimuth of sprag.

Figure 8 shows the influence of external torque $\mathrm{T}$ on the normal contact stiffness of sprag clutch. With increase of the external torque $\mathrm{T}$, the normal contact stiffness in $\mathrm{X}$ and $\mathrm{Y}$ axis direction increase non-linearly. When the external torque T is increased from 100 N.m to 1326.26 N.m, the normal contact stiffness in $\mathrm{X}$ axis direction will increase 1.44 times, and the normal contact stiffness in $\mathrm{Y}$ axis direction will increase 1.45 times.

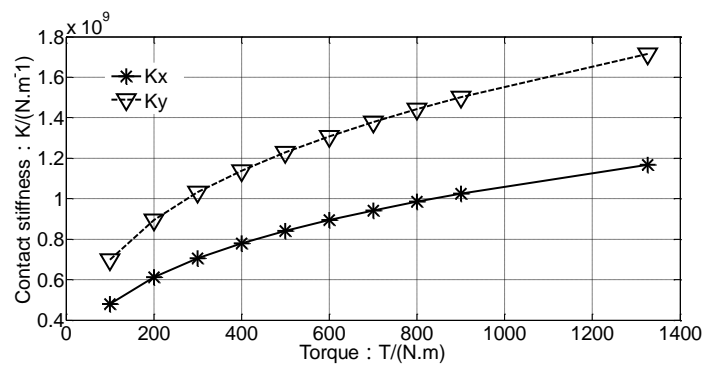

Figure 8 . The change rule of radial contact stiffness under different torque $\mathrm{T}$.

Figure 9 shows the influence of working speed of sprag one-way clutch on its normal contact stiffness. As it is shown that when the speed is increased from $1000 \mathrm{r} / \mathrm{min}$ to $7200 \mathrm{r} / \mathrm{min}$, the normal contact stiffness in $\mathrm{X}$ axis and $\mathrm{Y}$ axis remains the same value. It means that the effect of sprag centrifugal force can be ignored, when the contact stiffness of the clutch is calculated.

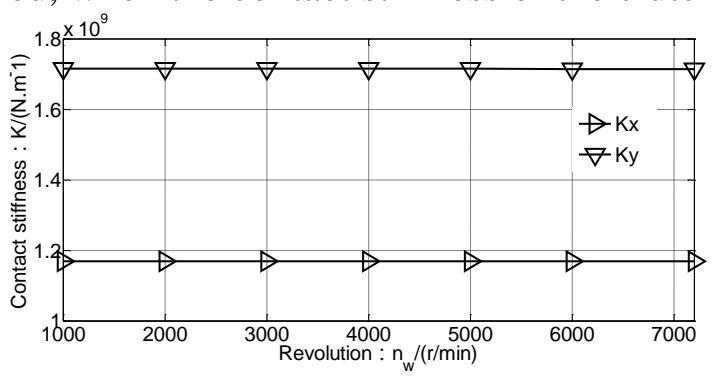

Figure 9. The change rule of radial contact stiffness under different revolution nw.

\section{Conclusions}

The smaller eccentricity e, the more sprag number of $\mathrm{Z}$ and the smaller radius difference $\mathrm{J}$ of between inner race and outer race will result larger stiffness.

(2) The working torque has the biggest influence on the contact stiffness of clutch. The greater the torque $\mathrm{T}$ is, the greater the normal contact stiffness in $\mathrm{X}$ and $\mathrm{Y}$ axial direction is. The rotational speed has very small influence on the normal contact stiffness.

(3) When there exists eccentricity e between the centers lines of the inner ring and outer ring, the normal load applied on each sprag is not equal. The normal contact load applied on the sprags 
located at the top and bottom of the $\mathrm{Y}$ axis direction is the maximum value, the two sprags located in $\mathrm{X}$ axis direction.

\section{Competing Interests}

The authors declare that there is no conflict of interests regarding the publication of this paper.

\section{Acknowledgments}

The authors thank the key research and development program in Hunan province (no.2015JC3007), the Basic Research on Digital Machining of Hard Processed Aeronautical Parts (no.2011CB706800), and general project in Hunan province department of education (no.15c1239) for financial support.

\section{Reference}

1. H.S. Han, K.H. Lee, S.H. Park, "Evaluation of the increased stiffness for the elastic coupling under the dynamic loading conditions in a ship," Engineering Failure Analysis, vol. 68, pp. 254262 (2016)

2. J.A. Abdo, "Investigation of contact stiffness and its relation to friction-induced noise and vibration," International Journal of Modeling and Simulation, vol. 26, No. 4, pp. 295-302 (2006)

3. F. R. Zhu and R. G. Parker, "Non-Linear Dynamics of a One-Way Clutch in Belt-Pulley Systems," Journal of Sound and Vibration, vol. 279, pp. 285-308 (2005)

4. D. Hu and J. W. Zu, "Effect of One-Way Clutch on the Nonlinear Vibration of Belt-Drive Systems with a Continuous Belt Model," Journal of Sound and Vibration, vol. 332, pp. 6472-6487 (2013)

5. D. Hu, "Periodic Responses of a Pulley-Belt System with One-Way Clutch under Inertia Excitation," Journal of Sound and Vibration, vol. 353, pp. 308-326 (2015)

6. G. J. Cheon, "Nonlinear Behavior Analysis of Spur Gear Pairs with a One-Way Clutch," Journal of Sound and Vibration, vol. 301, pp. 760-776 (2007)

7. T. Xu, G. G. Lowen, "A mathematical model of an over-running sprag clutch," Mechanism and Machine Theory, vol. 29, No. 1, pp. 11-23 (1994)

8. C. M. Chang, "Optimal design and control of systems with one-directional clutches for function generation," Madison: University of Wisconsin Madison (1996)

9. Y. Xue, N. L. Lu, X. Q. Qu, et al, "Research on torsional stiffness and contact consumptionenergy ofan arc-block-roller lower pair overrunning clutch," Journal of Harbin Engineering University, vol. 28, No. 7, pp.785-789 (2007)

10. X. C. Guo , Y. Su, H. L. Wang et al, "Calculation of the Torsion Stiffness of Overrunning Clutch in Wave Power Generation Device,"Journal of Mechanical Transmission, vol. 39, No. 2, pp.88-90 (2015)

11. Z. H. Liu, H. Z. Yan, Y. M. Cao, “A mathematical model for logarithmic spiral type sprag OneWay Clutch design and analysis," Journal of Central South University, vol. 22, No. 12, pp. 45974607 (2015)

12. Q. Chen, K. Huang, H. Zhao, etal, "Simulation and analysis of the model of calculating contact tangential stiffness between cylinders' joint interfaces by MATLAB," Appl Mech Mater 2012, vol.190, pp. 177-181 (2012) 
13. K. Huang, H. Zhao, Q. Chen, "Research on fractal contact model of cylinders' surface," Adv Tribol 2010, pp.548-553 (2010)

14. X. L. Zhang, Y. M. Huang, W. P. Fu, "Fractal model of normal contact stiffness between rough surfaces," Chin J Appl Mech 2000, vol.17, pp. 31-35 (2000) 